\title{
Carnosine and Its Role on the Erythrocyte Rheology
}

\author{
A. Seda Artis ${ }^{1}$ and Sami Aydogan ${ }^{2}$ \\ ${ }^{1}$ Physiology Department, Medical Faculty, Istanbul Medeniyet University \\ 2Physiology Department, Medical Faculty, Erciyes University \\ Turkey
}

\section{Introduction}

Erythrocytes are the most abundant cells (around 5 million $/ \mathrm{mm}^{3}$ ) in the body. The main function of these specialized cells is transport of oxygen $\left(\mathrm{O}_{2}\right)$ and mediation of carbondioxide $\left(\mathrm{CO}_{2}\right)$ production (Volpe, 1993). Mature erythrocytes have no capacity for cell division, protein synthesis, and mitochondrial-based oxidative reactions (Bunn, 1991; Benz, 2010). The erythrocyte possesses more membrane surface area than is needed to encase the volume of its cytoplasm. This allows for biconcave disc geometry. This shape can be stretched, twisted, distended, and compressed without permanent damage (Benz, 2010).

Erythrocytes are a highly specialized $\mathrm{O}_{2}$ carrier system in the body. More than $95 \%$ of cytoplasmic protein is hemoglobin (Telen \& Kaufman, 1999). Hemoglobin is the protein responsible for the oxygen-carrying capacity of erythrocytes. It provides the binding to $\mathrm{O}_{2}$ to heme, while keeping iron in the +2 oxidation state to assure the reversibility of this binding. Hemoglobin also facilitates exchange of $\mathrm{CO}_{2}$ (Nohl \& Stolze, 1998; Telen \& Kaufman, 1999). Under normal conditions, $95 \%$ of hemoglobin is saturated with oxygen in the lungs, whereas under physiologic conditions in peripheral blood stream only $25 \%$ of oxygenated hemoglobin becomes deoxygenated. Thus, the major fraction of oxygen bound to hemoglobin is recirculated with venous blood. The use of this fraction has been suggested for the treatment of oxygen deficiency. 2,3-Diphosphoglycerate (2,3-DPG) is a natural effector of hemoglobin. The binding affinity of hemoglobin for oxygen changes reversibly with the concentration of 2,3-DPG in the intracellular compartment. This compensates for changes in the oxygen pressure outside of the body, as the affinity of 2,3-DPG to oxygen is much higher than that of hemoglobin (Guyton \& Hall, 2000). The oxygen transport function of erythrocytes depends on the membrane being deformable and the components of the membrane play important roles in this function.

The erythrocyte membrane is composed of proteins (52\% in weight), lipids (40\%), and carbohydrates (8\%). Membrane elasticity depends on the structural interactions between the outer plasma membrane and the underlying protein skeleton (Desouky, 2009). The membrane comprises a lipid bilayer, integral membrane proteins and a membrane skeleton (Fig. 1). Integral proteins (glycophorin and Band 3 proteins) are tightly bound to the membrane through hydrophobic interactions in the bilayer (Lux, 1979; Mohandas, 1991; Mohandas \& Chasis, 1993). A filamentous network of proteins is anchored to the bilayer by 
the integral proteins. This network has three principal components: spectrin, actin, and protein 4.1 (Mohandas \& Chasis, 1993). The membrane skeleton proteins interact with the lipid bilayer and transmembrane proteins to give the red cell membrane its strength and integrity (Tse \& Lux, 1999). The peripheral membrane proteins are located on the cytoplasmic surface of the lipid bilayer and can be readily released from the membrane by simple manipulation of the ionic strength of the milieu or variation in the concentrations of other proteins (Mohandas \& Chasis, 1993). Besides control of cell shape, the cytoskeletal proteins have also roles in organization of specialized membrane domains, and attachment to other cells and substrates (Cimen, 2008).

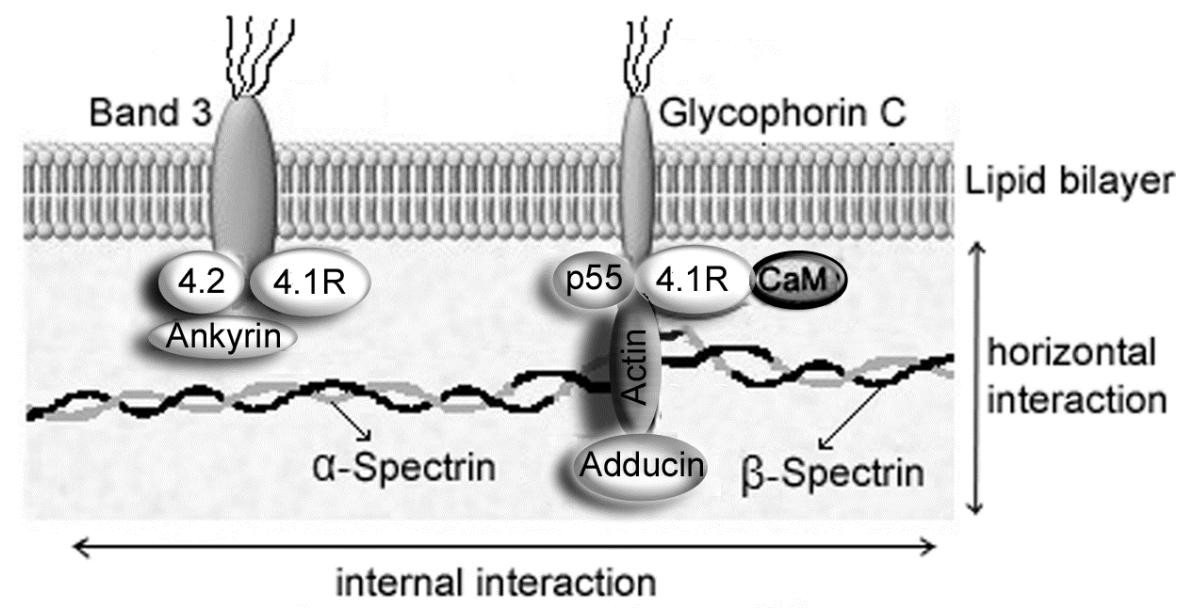

Fig. 1. The structure of erythrocyte membrane.

All lipids in the mature erythrocyte are found in the membrane bilayer and consist of phospholipid and cholesterol in 1.2:1 molar ratio. Approximately one-half of the fatty acids in the membrane are unsaturated (Telen \& Kaufman, 1999). Interestingly, outer surface lipids exchange freely with the plasma lipid compartment (Bunn, 1991). In addition, the structure of the lipid bilayer is critical to the cytoskeletal network organization within the erythrocyte (Smith et al., 2005). Disruption of the interaction between components of the red cell membrane skeleton at any contact point may cause loss of structural and functional integrity of the membrane. There accepted to be two types of interactions: vertical interactions between the membrane skeleton and the lipid bilayer, and horizontal interactions among components that form the membrane skeleton meshwork. The important links in the vertical interaction involve band 3, ankyrin, spectrin and protein 4.2. The critical horizontal interactions occur between the $\alpha$ and $\beta$ spectrins, $\beta$ spectrin and protein 4.1, and protein 4.1 and actin (Morris \& Lux, 1995).

Spectrin functions like a coiled spring able to stretch and snap back as the erythrocyte squeezes through capillaries, swells and shrinks, and is distorted by shear stresses. Spectrin attaches to the membrane via protein $4.1 \mathrm{R}$ and ankyrin. This confers sufficient tensile strength to withstand mechanical stresses and adequate flexibility to change shape as needed (Eber \& Lux, 2004). The flexible, biconcave shape enables erythrocytes to squeeze through narrow capillaries. The erythrocytes need to endure both the micro and 
macrocirculatory environment. The rheological properties of erythrocytes microstructure play important roles in microcirculation, and also blood flow in large arteries. Microscopic mechanisms can be connected to the macroscopic behaviors of the blood and transferred by means of a blood viscosity model based on blood structure to the macroscopic behaviors of the blood (Yilmaz \& Gundogdu, 2008).

Glucose, the only fuel utilized by erythrocytes, is primarily metabolized via anaerobic glycolysis. Following facilitated diffusion, glucose is immediately converted to glucose- 6 phosphate. Approximately $80-90 \%$ percent is then converted to lactate via the glycolytic pathway. The remaining $10 \%$ undergoes oxidation via the pentose phosphate shunt. Glucose metabolism effectively maintains glutathione in the reduced form thereby protecting hemoglobin sulfhydryl groups and erythrocyte membranes from oxidation. A significant portion of the adenosine triphosphate (ATP) generated by glycolysis is spent in operating the sodium potassium pump necessary to reserve the cytoplasmic ionic milieu thus preventing colloidal osmotic lysis. In addition, some metabolic energy is expended on maintenance and repair of the red cell membrane (Bunn, 1991).

\section{Erythrocytes, oxidative stress and aging}

Mature, circulating erythrocytes have a finite life span. Each day, less than 1\% of these cells are destroyed and replaced by virtually identical numbers of new cells. The molecular mechanism that determines removal of aged or damaged erythrocytes from the circulation remains unknown, but probably involves recognition of senescence antigens by phagocytes (Volpe, 1993). It has proposed that the major senescence antigen in aged erythrocytes is derived from the band 3 protein, the main transmembrane glycoprotein in erythrocytes. Other possible mechanisms for erythrocyte aging include mechanical fatigue, ATP depletion, calcium accumulation, and the generation of reactive oxygen species (ROS) (Feher et al., 2006). Erythrocytes experience continuous oxidative insult by being exposed to endogenous and exogenous ROS. ROS, which damage proteins and initiate lipid peroxidation, can be generated either inside erythrocytes through the hemoglobin oxidation pathway or outside. Although the erythrocyte contains an extensive antioxidant defense system, oxidative damage of membrane proteins and lipids contributes to the senescence of normal erythrocytes and results in a shorter life span for pathological cells.

The major source of intracellular ROS in the erythrocyte is autoxidation of oxyhemoglobin, which generates superoxide and produces hydrogen peroxide $\left(\mathrm{H}_{2} \mathrm{O}_{2}\right)$. Catalase and glutathione peroxidase (GSHPx) scavenge most of the $\mathrm{H}_{2} \mathrm{O}_{2}$ generated in the cells. Degradation of the heme moiety takes place in conjunction with the reaction of $\mathrm{H}_{2} \mathrm{O}_{2}$ with hemoglobin. In addition, even small concentration of $\mathrm{H}_{2} \mathrm{O}_{2}$ generated during the autoxidation of oxyhemoglobin contributes to heme degradation. Heme degradation is, therefore, expected to take place in the erythrocyte when the antioxidant enzymes are not able to eliminate all the $\mathrm{H}_{2} \mathrm{O}_{2}$ (Snyder et al., 1985; Prokopiva et al., 2000; Feher et al., 2006).

One of the well established mechanisms of mechanical impairment of the erythrocyte is oxidative damage (Fig.2). Erythrocyte deformability is determined by cellular geometry, cytoplasmic viscosity of erythrocyte (hemoglobin concentration), and viscoelastic properties of the erythrocyte membrane. Membrane viscoelasticity is in turn determined by erythrocyte membrane skeleton, which is mainly a spectrin network attached to the integral proteins. 
Oxidative reactions that start in the lipid components (i.e. lipid peroxidation) lead to the formation of cross-linkages within the membrane skeletal proteins or hemoglobin, increasing membrane viscosity. Additionally, oxidative damage may affect transport processes through the erythrocyte membrane, affecting the cell geometry and cytosolic viscosity (Feher et al., 2006; Aydogan et al., 2008).

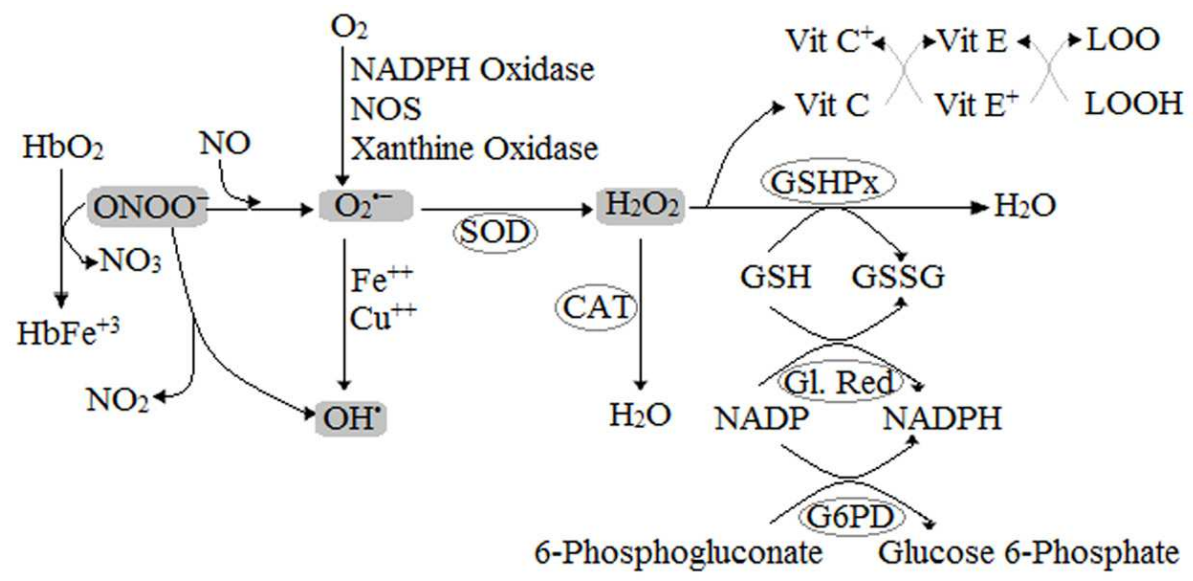

Fig. 2. Free radical metabolism in human erythrocytes.

The general consensus appears to be that the aging process is multifactorial and that ROS are a contributing factor. Particularly two phenomena are of particular concern: the deleterious effects of ROS and the formation of reactive carbonyl compounds related to the glycation reaction, involved in the acceleration of molecular and tissue aging processes. The ROS theory of erythrocyte aging has been widely accepted, yet it lacks direct supporting evidence, and the extent of ROS contribution remains uncertain.

The balance between ROS production and antioxidant defences determines the degree of oxidative stress. Unfortunately, the activity of these systems declines during aging, so the consequences of this stress include modification to to cellular components. Following the ROS mediated oxidation of sugar and membrane lipids through a complex and still unclear cascade of reactions. Carbonyl compounds are very reactive small molecules which can be considered a key oint in the propagation and amplification of the aging process. Carbonyl reactive compounds are able to form adducts commonly known as CO-proteins (proteins bearing carbonyl groups) with structural proteins lipoproteins, enzymes and with DNA, causing alterations in their biological activity thrugh a whole of chemical raction steps in all known as glycation reaction.

Glycation (or glycosylation) reaction is a reaction between reducing sugars, or other carbonyl group bearing molecules, and free amino groups of protein, leading to the formation of abnormal products, namely Advanced Glycation End Products (AGEs, crosslinked proteins). AGEs are very toxic for the cells, as they are very rich in double bonds which can interact irreversibly with biological substrates, leading to a loss of their physiological function. The result of cross-linking is a loss of physiological function, loss of genome information, and consequently senescence. 


\section{General effects of carnosine}

L-Carnosine (B-alanyl-L-histidine) is a naturally occurring dipeptide and present in food. It is also commonly present in mammalian tissues. Carnosine is found naturally in the body. The highest concentrations are present in long-lived cells; particularly in skeletal muscles, followed by the heart, cerebellum and brain. No carnosine is detectable in plasma, liver kidney, and lung. It is formed by carnosine synthetase enzyme. It is kept in equilibrium by carnosinase enzyme. However its levels within the tissues decline with age.

Its proper function still remains unknown, although many properties have been proposed including physiological buffer (helps maintenance of the $\mathrm{pH}$ balance in the muscles in heavy exercise), wound healing agent, antioxidant (prevents the modification of biomacromolecules thereby keeping their native functionality under oxidative stress), freeradical and active sugar molecule scavenger (prevents glycation and carbonylation of proteins), heavy metal chelator (especially copper and zinc), immunomodulator and antitumor agent (e.g. suppresses of proinflammatory and carcinogenic cytokine IL-8), and anti-aging compound (Aruoma et al., 1989; Quinn et al., 1992; Gariballa \& Sinclair, 2000).

Carnosine is a naturally occurring antioxidant that is also an anti-glycating agent. It has the ability to suppress Advanced Glycation End Products (AGEs) and formation of reactive oxygen species (ROS). In a remarkable series of experiments, scientists have shown that carnosine rejuvenates cells as they approach senescence (McFarland \& Holliday, 1994, 1999). As shown by experiments on fibroblast cultures, carnosine retains youthful appearance and growth patterns. Fibroblasts that went through many rounds of division, known as latepassage cells, displayed a disorganized, irregular appearance before ceasing to divide. However fibroblasts cultured with carnosine lived longer, retaining youthful appearance and growth patterns. But, interestingly, when they transferred the fibroblasts back to a medium lacking carnosine, the signs of senescence quickly reappeared. The scientists switched late-passage fibroblasts back and forth several times between the culture media. They consistently observed that the carnosine culture medium restored the juvenile cell phenotype within days, whereas the standard culture medium brought back the senescent cell phenotype (McFarland \& Holliday, 1994, 1999). Another group tested the effect of carnosine on life span and indicators of senescence in senescence-accelerated mice. Carnosine added to drinking water distinctly improved the appearance of the aged mice, whose coat fullness and color remained much closer to that of young animals The carnosine medium also increases life span, even for old cells. In the same study carnosine extended the life span of the treated mice by $20 \%$ on average, compared to the control mice. Carnosine did not alter the 15 month maximum life span of the senescence-accelerated mice strain, but it did significantly raise the number of mice surviving to old age (Boldyrev et al., 1999; Yuneva et al., 1999).

The current knowledge about the mechanisms involved in the aging process and the defense mechanisms are described in some experiments. Particularly, two phenomena are of particular concern: The deleterious effects of reactive oxygen species and the formation of reactive carbonyl compounds related to the glycation reaction, involved in the acceleration of molecular and tissue aging processes. The powerful and effective action of carnosine is performed against all the elements that triggered the aging process and against all the phenomena that contribute to its propagation and amplification (Hipkiss \& Chana, 1998; Tamba \& Torreggiani, 1999). 
The anti-aging actions of L-carnosine may be summarized as follows (Fig.3): Carnosine stops the oxidative damage acting as an antioxidant agent, a ROS scavenger agent, metal ions chelating agent and by expressing superoxide dismutase (SOD)-like activity. It inhibits the glycation reaction, by quenching carbonyl compounds and AGEs. It also prevents the cross-linking of the macromolecules and promotes modification in enzyme-mediated protein degradation.

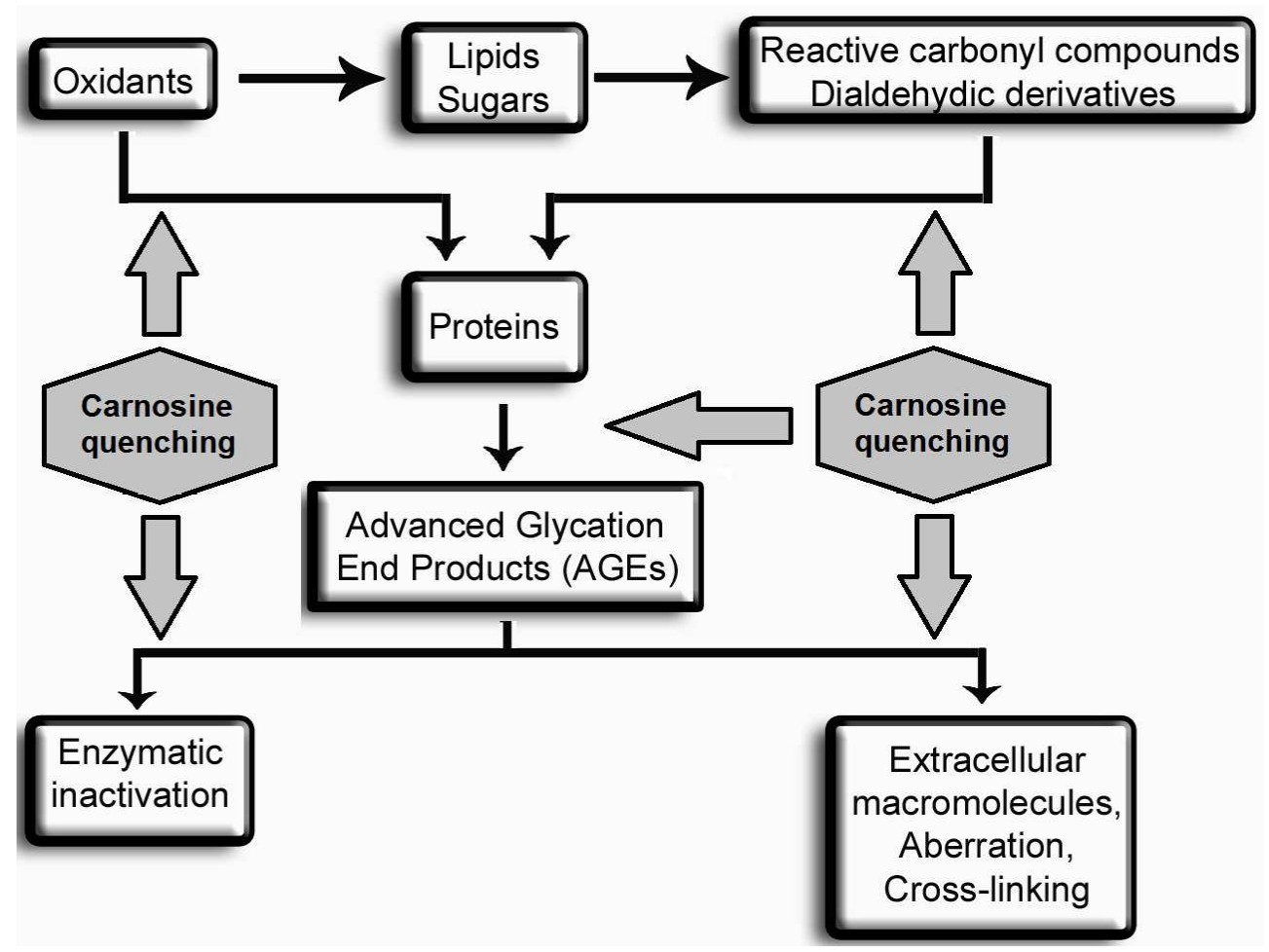

Fig. 3. The summary of sites of intervention of L-carnosine as anti-aging molecule.

Carnosine is widely believed to be an antioxidant which stabilizes and protects the cell membrane, and an oxygen free radical-scavenger (Kohen et al., 1988; Aruoma, Laughton et al., 1989; Boldyrev, Song et al., 1999). Specifically, as a water-soluble free radical scavenger it prevents lipid peroxidation within the cell membrane (Tamba \& Torreggiani, 1999). It is thought to be a natural counterpart to lipid-soluble antioxidants such as vitamin E. Many antioxidants prevent free radicals from entering the tissues, but have no effect after this first line of defense is broken. Carnosine is not only effective in prevention, but it is also active after free radicals react to form other dangerous compounds. So, it protects the tissues from these damaging 'second-wave' chemicals. For example, it blocks a highly reactive lipid peroxidation end-product called malondialdehyde (MDA) (Kohen et al., 1988; Aruoma et al., 1989; Hipkiss et al., 1997; Hipkiss et al., 1998; Boldyrev, Song et al., 1999). MDA, if left uncontrolled, can cause damage to lipids, enzymes and DNA, and plays a part in the 
process of atherosclerosis, joint inflammation, cataract formation, and aging in general. Carnosine, by reacting and inactivating MDA, (Hipkiss et al., 1997) sacrifices itself in order to protect the amino acids on the protein molecule (Andrea et al., 2005). It has also the ability to reduce concentrations of thiobarbituric acid reactive substances (TBARS). Interacting with aldehydic lipid oxidation products, carnosine protects biological tissues from oxidation, since aldehydes can form adducts with DNA, proteins, enzymes, and lipoproteins, causing harmful alterations in their biological activity (Burcham et al., 2002). Many studies have demonstrated at tissue, cell and organel levels, that carnosine may prevent peroxidation of many model membrane system and also cell membrane, including erythrocytes (Boldyrev et al., 1997). Carnosine inhibits lipid oxidation by a combination of free radical scavenging and metal chelation. It has an ability to chelate prooxidative metals, such as copper, zinc and toxic heavy metals (lead, mercury, cadmium, nickel). Carnosine, as a dietary supplement, seems to have all the same chelating properties as EDTA (Hipkiss, 2005).

Carnosine can claim different properties performed at different steps of the whole aging process. Specifically, as a water-soluble free radical scavenger carnosine prevents lipid peroxidation within the cell membrane. Carnosine is not only effective in prevention, but it is also active after free radicals react to form other dangerous compounds. So, it protects the tissues from these damaging "second-wave" chemicals. It stops the oxidative damage acting as an antioxidant agent, a ROS scavenger agent, metal ions chelating agent and by expressing a superoxide dismutase (SOD) - like activity. It inhibits the glycation reaction, by quenching carbonyl compounds and AGEs. It prevents the cross-linking of the macromolecules (Hipkiss \& Chana, 1998; Tamba \& Torreggiani, 1999).

Age-related conditions that carnosine may be useful for: diabetes and its complications, neurological degeneration (Alzheimer's, Parkinson's, epilepsy, depression, schizophrenia, mild cognitive impairment, dementia and stroke), autistic spectrum disorders, cellular senescence in general cross-linking of the eye lens (cataracts), cross-linking of skin collagen (skin ageing), formation of AGEs, accumulation of damaged proteins, muscle atrophy, brain circulation deficit (stroke), and cardiovascular conditions (Boldyrev et al., 1997; Hipkiss, 2005).

One of the cardinal processes of aging, apart from the free-radical damage, is the process of glycosylation (or glycation). During normal metabolism, sugar aldehydes may react with the amino acids on the protein molecule, resulting in the formation of AGEs. AGEs are very toxic for the cells, which can react irreversibly with biological substrates leading to a loss of their physiological functions. Carnosine inactivates not only aldehydes and ketones, therefore reducing protein glycosylation and the formation of AGEs; but also already formed AGEs. Normally, AGEs are removed by scavenging macrophages which carry special receptors called RAGEs. Carnosine facilitates this process of elimination, by helping macrophages to better recognize the AGE molecules. Because of its anti-glycosylation actions, carnosine may be useful in treating or preventing diabetic complications such as cataract, neuropathy and kidney failure (Hipkiss \& Chana, 1998).

With regard to oxidative modifications and erythrocyte demise, recent insights come from studies on their senescence suggesting that they can undergo a sort of apoptosis (Boas et al., 1998; Daugas et al., 2001). In particular, the apoptosis of erythrocytes was called eryptosis or 
erythroptosis depending on the injury pathway. The biological meaning and relevance of erythrocyte senescence and apoptosis, characterized by glycophorin A loss or phosphotidyl serine externalization respectively. However, although these are mainly referred to as critical events responsible for erythrocyte removal at the end of their life span, that subject is still a matter of debate (Daugas et al., 2001; Head et al., 2005; Pietraforte et al., 2007). For instance, the plethora of changes occurring in senescent and apoptotic erythrocytes under oxidative/nitrosative stress definitely comprises even biophysical changes, e.g. the loss of cell plasticity with impaired deformability associated with changes of cytoskeletal network assembly (Marchesi, 1985; An et al., 2002).

\section{Effects of carnosine on erythrocyte rheology}

The rheological properties of erythrocytes play important roles in microcirculation, and also blood flow in large arteries. Microscopic mechanisms can be connected to the macroscopic behaviors of the blood and transferred by means of a blood viscosity model based on blood structure to the macroscopic behaviors of the blood (Yilmaz \& Gundogdu, 2008).

"Deformability" is the term generally used to characterize the erythrocyte's ability to undergo deformation during flow (Mohandas \& Chasis, 1993). The deformation response of an erythrocyte to fluid forces is a complex phenomenon that depends on a number of different cell characteristics including membrane material properties (Lux, 1979), cell geometry, and cytoplasmic viscosity (Mohandas, 1991). Erythrocyte deformability is an important determinant of blood rheology, either in bulk flow conditions or microcirculation. Normal erythrocyte deformability is essential for proper tissue perfusion and oxygenation, as well as the normal survival of erythrocyte in the circulation.

Blood is a non-Newtonian fluid and its viscosity is therefore variable at any given temperature, depending on the shear rate. At low shear rate, erythrocytes can aggregate and form one-dimensional stacks-of-coins-like rouleaux or three-dimensional aggregates. This is because of the electrostatic repulsion of erythrocyte overcome by the present macromolecules which aggregate the cells. The process is reversible and particularly important in the microcirculation, since such rouleaux or aggregates can dramatically increase effective blood viscosity. Erythrocytes may also exhibit reduced deformability and stronger aggregation in many pathological situations, such as heart disease, hypertension, diabetes, malaria, and sickle cell anemia (Popel \& Johnson, 2005).

The resistance of erythrocytes to hemolytic action is an integral parameter characterizing their integrity and viability as well as a criterion of their physiologically native state (Arzumanyan et al., 2008). Various factors can cause hemolysis of the erythrocytes, including a decreased ambient osmotic pressure, decreased $\mathrm{pH}$, and oxidants (Ivanov, 1999; Pribush et al., 2002).

Oxidative damage in erythrocyte is one of the well established mechanisms of mechanical impairment. Erythrocytes are exposed to oxygen radicals continuously generated via the autoxidation of hemoglobin. In addition erythrocytes have relatively high levels of polyunsaturated fatty acids (PUFA), which are good substrates for peroxidation reactions. So due to the high iron content, relatively less antioxidant activities and high PUFA content the erythrocyte has a limited antioxidant defence. 
Carnosine act as an antioxidant as well as a free-radical scavenger, which can protect and stabilize the cell membrane from non-enzymatic glycosylation and oxidation (Tamba \& Torreggiani, 1999; Hipkiss \& Brownson, 2000). In addition, carnosine also prevents protein glycation by using an enzyme that catalyses the splitting of interior peptide bonds in a protein (Quinn et al., 1992). Furthermore, the ability of carnosine to disintegrate the readily glycated protein was observed through the hydration and unfolding of deleterious reactions, such as the cross-linking of proteins (Seidler et al., 2004).

The significant role of L-carnosine in maintaining erythrocyte physiology under oxidative stress has been demonstrated previously by Aydogan et al (Aydogan et al., 2008). In that study blood from 3 and 10 months old rats had been used. L-carnosine improved erythrocyte deformability significantly in healthy erythrocytes independent of age. L-carnosine also significantly improved deformability in damaged erythrocytes as well both in the young and old rats (Fig.4). This study provided the first evidence for importance of carnosine in maintaining normal erythrocyte properties and to protect them from oxidative damage induced by $\mathrm{H}_{2} \mathrm{O}_{2}$ administration under in vitro conditions. This observation was in favor of the idea that L-carnosine supplementation might be used to improve erythrocyte quality.
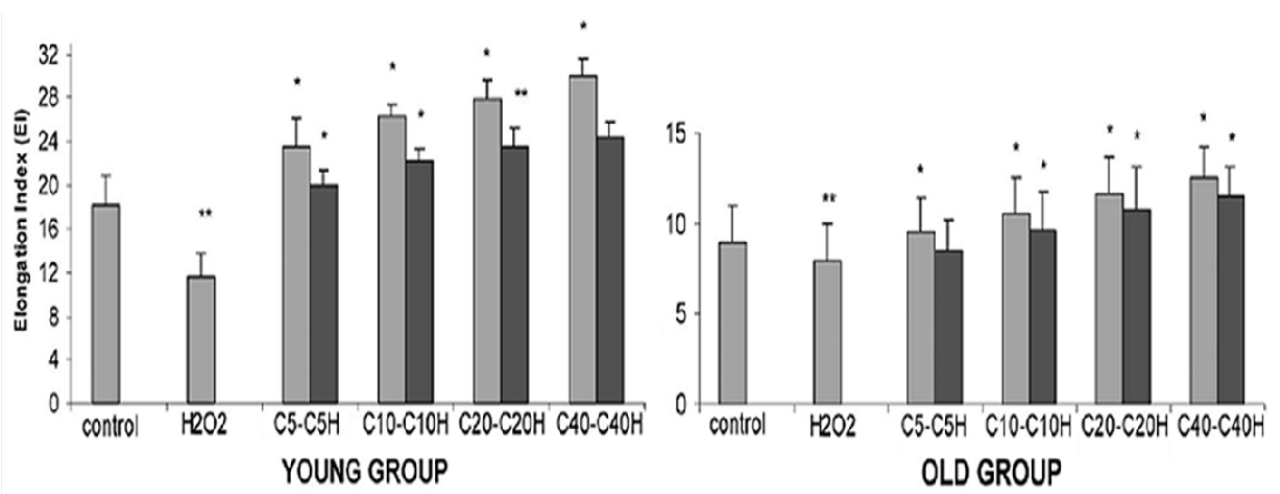

Fig. 4. Erythrocyte elongation indexes (EI) in control, treated with $\mathrm{H}_{2} \mathrm{O}_{2}$ and different concentrations of L-carnosine $(5-40 \mathrm{mM})$ groups. Values are presented as mean $\pm \mathrm{SD}, n=10$ for each group, ${ }^{*} p<0.05$ when compared to $\mathrm{H}_{2} \mathrm{O}_{2},{ }^{*} p<0.05$ when compared to control. " $\mathrm{C}$ " and " $\mathrm{H}$ " respectively indicates L-carnosine and $\mathrm{H}_{2} \mathrm{O}_{2}$ applications. The numbers following the letter " $\mathrm{C}$ " show carnosine concentrations. Reproduced from Aydogan et al. 2008 (Aydogan et al., 2008).

Another study from the same group showed that in vivo carnosine supplementation can be used to protect the erythrocytes from oxidative or peroxidative damage (Yerer et al., 2010). Sodium nitroprusside (SNP), which is a potent hypotensive agent, had been used to induce oxidative/nitrosative damage. Nitric oxide (NO) is a signaling molecule of major importance modulating not only the function of the vascular wall but also that of blood cells, such as platelets and leukocytes. The synthesis of NO in the circulation has been attributed mainly to the vascular endothelium. Erythrocytes have been demonstrated to carry a non-functional nitric oxide synthase (NOS) and due to their huge hemoglobin content have been assumed to metabolize large quantities of NO. However, more recently erythrocytes have been identified to reversibly bind, transport, and release NO within the 
cardiovascular system. This function of erythrocytes on NO metabolism also reflects the importance of oxidative damage via peroxinitrite. Oxidative and nitrosative damage to membrane erythrocyte membrane leads to the impairment of biorheological properties of the blood (Yerer et al., 2010). It is already known that NO increased either in pathological conditions and treatments with NO derivatives increase lipid peroxidation in the membrane of the erythrocytes (Yerer et al., 2004). When L-carnosine was given to rats intraperitoneally before the induction of oxidative damage by sodium nitroprusside, this prevented the increase in malondialdehyde (MDA), glutathione peroxidase (GSH-Px), and superoxide dismutase (SOD) (Fig.5A,B,C) activities. The changes in erythrocyte elongation indexes were in parallel as expected with these observations (Fig.5D). Carnosine application also significantly increased the erythrocyte elongation indexes that had already decreased by sodium nitroprusside (Yerer et al., 2004).

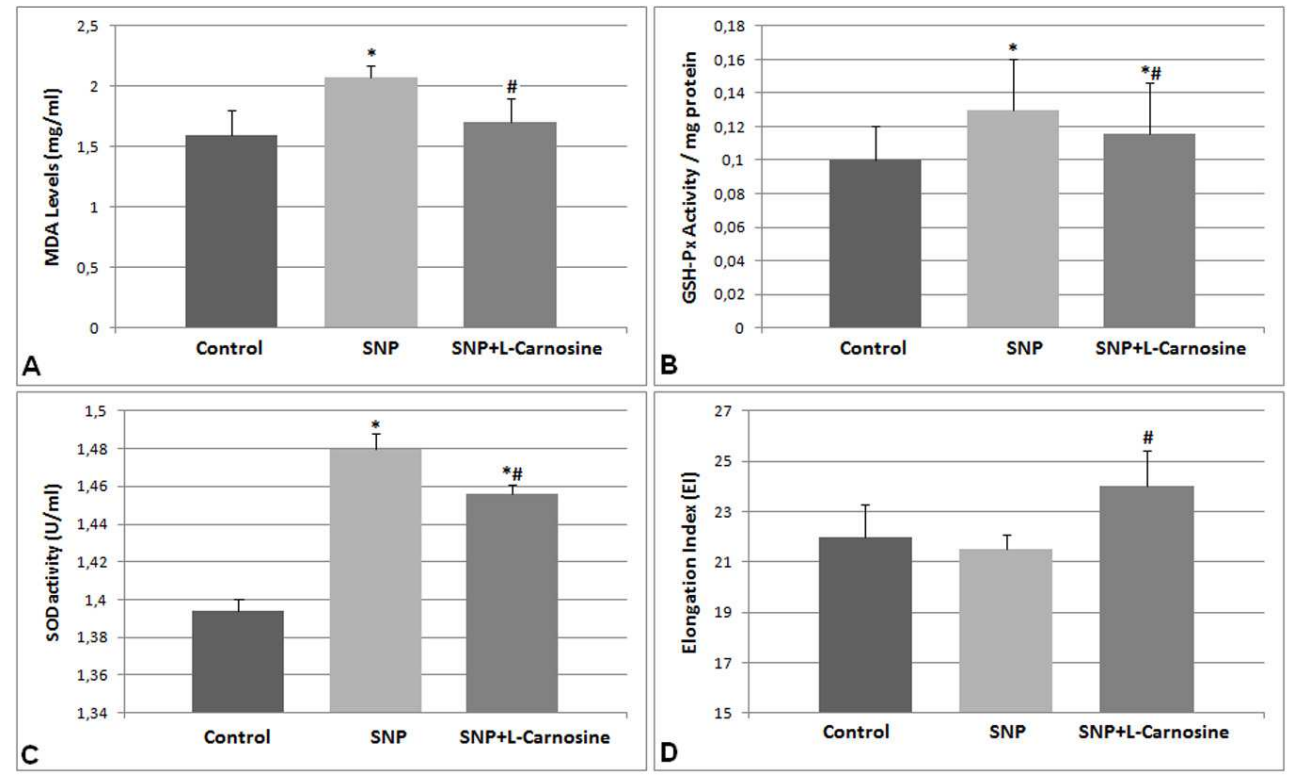

Fig. 5. A: Malondialdehyde (MDA) levels reflecting the lipid peroxidation. B: Glutathione peroxidase (GSH-Px) enzyme activity. C: Superoxide dismutase (SOD) enzyme activity. D: Elongation indexes (EI) reflecting erythrocyte deformability. *: significantly different from the control, \#: significantly different from the sodium nitroprusside (SNP) group.

Reproduced from Yerer et al 2010 (Yerer et al., 2010).

Similarly Arzumanyan et al have demonstrated that homocysteic acid (HCA) provokes oxidative stress in erythrocytes and decreases their hemolytic resistance, whereas the natural antioxidant carnosine protects erythrocytes from its toxic effect (Arzumanyan et al., 2008). Actually this should be taken into account when assessing the states of patients with chronic hyperhomocysteinemia. Homocysteine and the product of spontaneous homocysteine oxidation, namely HCA, are important risk factors for neurodegenerative and cardiovascular diseases (Jacobsen, 1998). The prooxidant effects of homocysteine and HCA on cell structures possibly occur via both the glutamate receptors (Vladychenskaya et al., 
2006) and the activation of $\mathrm{NO}$ synthase or inhibition of $\mathrm{Na}^{+} / \mathrm{K}^{+}$ATPase (Arzumanyan et al., 2008). It is known that erythrocytes are capable of accumulating homocysteine and excreting it into the extracellular medium (Preibisch et al., 1993; Schlussel et al., 1995). Changes in the ambient osmotic pressure, $\mathrm{pH}$, and oxidant levels are among the various factors causing hemolysis of erythrocytes. The resistance of erythrocytes to hemolytic action is an integral parameter characterizing their integrity and viability as well as a criterion of their physiologically native state. The investigators determined the level of free radicals in erythrocytes flow cytometrically and observed again that carnosine has a preventive effect on the harmful effects of HCA. The group tested any possible effects on erythrocytes by both osmotic and acid hemolysis (caused by hydrochloric acid) methods. The maximal number of cells hemolyzed osmotically by the end of the process remained the same. HCA increased the rate of acid erythrocyte hemolysis by $18-25 \%$, whereas carnosine decreased the hemolysis rate to $80 \%$ of the control value irrespective of the presence or absence of HCA in the sample. The data from the study of Arzumanyan et al provided evidence for: (a) the preincubation of erythrocytes with homocysteic acid (HCA) considerably increases the hemolysis rate, (b) whereas carnosine prevents the hemolytic effect of HCA. The authors claim that the protective effect of carnosine had a pronounced dose-dependent manner.

Hemorheological properties are easily modified by glucose-induced oxidation and glycation. Carnosine prevents protein glycation by using an enzyme that catalyses the splitting of interior peptide bonds in a protein (Quinn et al., 1992). Furthermore, the ability of carnosine to disintegrate the readily glycated protein was observed through the hydration and unfolding of deleterious reactions, such as the cross-linking of proteins (Seidler et al., 2004). Another hemorheological in vitro study, conducted by Nam et al. on erythrocytes incubated in glucose-rich media, revealed the beneficial effects after addition of carnosine (Nam et al., 2009). In that study erythrocytes were incubated in glucose-rich media with different concentrations of carnosine. Also, defective erythrocytes due to hyperglycemia were incubated in autologous plasma with different concentrations of carnosine. When there was no carnosine in glucose-rich solution, both the erythrocyte deformability and aggregability significantly decreased. The degree of impairment in erythrocyte deformability and aggregability was proportional to the glucose-concentration. However, through the addition of carnosine to glucose solutions, the impairment of erythrocyte deformability and aggregability gradually diminished. To examine the rejuvenating function of carnosine, same researchers incubated erythrocytes, which were exposed to hyperglycemia-associated oxidative stress, in plasma that was supplemented with carnosine. As the carnosine concentration increased, the deformability of impaired erythrocytes slightly increased. Nam et al. also suggested that by increasing the incubation time, the deformability could be further increased. However, an increase in the carnosine concentration could cause echinocytic change in the shape of erythrocytes due to hyperosmolarity. During the same experiment researchers also investigated the aggregability of the erythrocytes. As the carnosine concentration increased in plasma, the aggregation index (AI) of impaired erythrocytes increased in a concentration-dependent manner. In addition, the increase in erythrocyte aggregability was also strongly dependent upon the incubation time, and the effect of carnosine was more prominent on aggregation than deformability. Thus, longer incubation periods for erythrocytes might increase erythrocyte aggregability up to the value of the control. The results of mentioned study reveal that the presence of carnosine effectively prevented rheological alterations due to glucose-induced oxidation and glycation in a concentration-dependent manner (Nam et al., 2009). 
Diabetes mellitus is a disease characterized by insulin deficiency. It is known that oxidative stress plays an important role in physiopathology of chronic complications in diabetes. Since the structure of carnosine closely resembles that of the preferred glycation sites in proteins, the glycated sites may be occupied by carnosine, which, in turn, results in the rejuvenation of hemorheological characteristics. It is commonly known that most anti-oxidants prevent free radicals from binding the proteins but have no effect after protein binds with the free radicals. Yapislar and Aydogan have investigated the effects of experimental diabetes on erythrocytes in rats (Yapislar \& Aydogan, 2011). In that in vivo study erythrocyte deformability indexes and NO levels were decreased and MDA levels were found to be increased in diabetic group. Erythrocyte deformability abilities are reduced as a result of lipid peroxidation in erythrocyte membrane under diabetic condition (Yapislar \& Aydogan, 2011; Nam et al., 2009). On the other hand, decrease in endothelium nitric oxide production might be responsible in endothelial dysfunction seen in diabetic vascular complications. NO is thought to cause oxidative and nitrosative damage to the erythrocyte. $\mathrm{NO}$ is a crucial component for erythrocytes which maintains different biological functions within the circulation. However, the excessive amounts of NO can trigger the oxidative and nitrosative damage especially to erythrocytes which leads to the impaired tissue perfusion (Yerer et al., 2010; Yapislar \& Aydogan, 2011). Recent findings on the ability of carnosine to interact with guanylate cyclase heme also reflect the importance of this molecule in endogenous regulation of this enzyme over heme molecule (Severina et al., 2000). The data from the above mentioned study (Yapislar \& Aydogan, 2011) revealed that carnosine application to diabetic rats significantly reversed the erythrocyte deformability and reduced the lipid peroxidation under diabetic conditions (Fig.6).

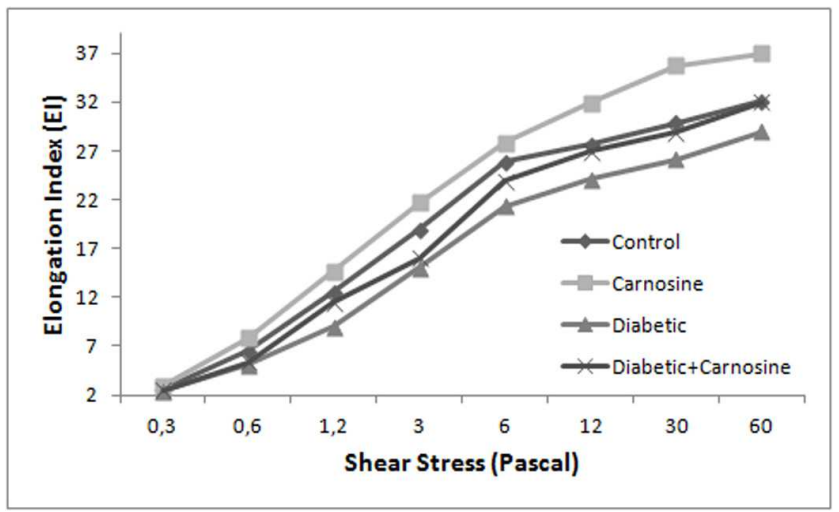

Fig. 6. Elongation indexes reflecting erythrocyte deformability after L-carnosine application in experimental diabetic rats at different shear stress rates. Reproduced from Yapislar and Aydogan 2011(Yapislar \& Aydogan, 2011).

Aydogan et al. also recently studied deformability, aggregability, and osmotic fragility properties of healthy rat erythrocytes following incubation with L-carnosine (Aydogan et al., 2010) (article submitted). The data revealed that L-carnosine has a dose dependent positive effect on RBC deformability and aggregability. In the presence of carnosine, erythrocytes showed also an increased ability to resist hemolysis. This dipeptide appears to be rejuvenating or to improve erythrocyte quality and mechanical properties. 


\section{Conclusion}

Carnosine is a natural and nontoxic compound. It has a high bio-availability and is lack of side effects. The beneficial properties of this dipeptide appear to be rejuvenating or to improve quality and mechanical properties of healthy erythrocytes. L-carnosine supplemention also can be used to protect them from several conditions or damages in survival of RBC in the circulation. Furthermore, use of carnosine after the pathology has occurred also helps to improve the deteriorated hemorheological status. These effects can be related to its antioxidant, free-radical scavenger, antiglycation and buffering properties.

L-carnosine helps healthy erythrocytes to fight agains oxidative stress and improve their survival in microcirculation. Supplementation with carnosine has rejuvenating effects on the healthy erythrocytes. It protects erythrocytes from oxidative stress occurring due to exposure to harmful conditions, including nitrosative stress, and glucose-induced oxidative stress and glycation. These beneficial effects of L-carnosine are dose and possibly incubation time dependent. Besides, damaged erythrocytes, as in the condition of experimental diabetes, also seem to improve following carnosine application. Carnosine performs an apparent rejuvenating function for hemorheologically damaged cells.

L-carnosine therefore seems to have crucial biological functions on erythrocytes which need to be identified with further investigations. In the light of the above mentioned studies in the literature it can be suggested that:

a. Carnosine can be used as for maintaining normal erythrocyte properties in healthy subjects and to improve the erythrocyte quality and survival.

b. L-carnosine can be used as a supplement in diabetes. It can recover microvascular circulation problems by increasing erythrocyte deformability, can reduce the risk of atherosclerosis and cardiovascular disease in diabetes by increasing NO levels, can protect cells and tissues against harmful effect of lipid peroxidation by decreasing lipid peroxidation and can be used as a multi-functional antioxidant in the treatment of diabetes mellitus to prevent the complications of diabetes.

c. There is a possibility of L-carnosine to be used for its protective effects on nitric oxide donor pharmaceutical damages in the circulation.

d. L-carnosine might be used as a pharmaceutical drug for treatment of malignant tumors, sepsis, asthma, migraine, i.e. pathologies which are associated with increasing the NO levels.

e. Moderate concentrations of carnosine might be further explored as potential therapeutic agents for pathologies that involve hemorheological modification.

f. Further experiments are in progress and it is expected that carnosine supplementation will become much more widespread during the next years.

\section{References}

An, X.; Lecomte, M.C.; Chasis, J.A.; Mohandas, N. \& Gratzer, W. (2002). Shear-response of the spectrin dimer-tetramer equilibrium in the red blood cell membrane. J Biol Chem, Vol.277, No.35,pp.31796-31800.

Andrea, R.G.; Andrea, C.; Paolo, R. \& Gianfranco, B. (2005). Carnosine and carnosine-related antioxidants: A review. . Curr Med Chem., Vol.12, No.20,pp.2293-2315. 
Aruoma, O.I.; Laughton, M.J. \& Halliwell, B. (1989). Carnosine, homocarnosine and anserine: could they act as antioxidants in vivo? Biochem J, Vol.264, No.3,pp.863-869.

Arzumanyan, E.S.; Makhro, A.V.; Tyulina, O.V. \& Boldyrev, A.A. (2008). Carnosine protects erythrocytes from the oxidative stress caused by homocysteic acid. Dokl Biochem Biophys, Vol.418,pp.44-46.

Aydogan, S.; Artis, A.S. \& Basaran, E. (2010). A possible new role of L-carnosine as rejuvenating agent for improvement of erythrocyte quality and mechanical properties. 3rd Antiaging Congress. Ankara, Turkey June 2010, pp.17.

Aydogan, S.; Yapislar, H.; Artis, S. \& Aydogan, B. (2008). Impaired erythrocytes deformability in $\mathrm{H}(2) \mathrm{O}(2)$-induced oxidative stress: protective effect of L-carnosine. Clin Hemorheol Microcirc, Vol.39, No.1-4,pp.93-98.

Benz, E.J., Jr. (2010). Learning about genomics and disease from the anucleate human red blood cell. J Clin Invest, Vol.120, No.12,pp.4204-4206.

Boas, F.E.; Forman, L. \& Beutler, E. (1998). Phosphatidylserine exposure and red cell viability in red cell aging and in hemolytic anemia. Proc Natl Acad Sci U S A, Vol.95, No.6,pp.3077-3081.

Boldyrev, A.; Song, R.; Lawrence, D. \& Carpenter, D.O. (1999). Carnosine protects against excitotoxic cell death independently of effects on reactive oxygen species. Neuroscience, Vol.94, No.2,pp.571-577.

Boldyrev, A.A.; Stvolinsky, S.L.; Tyulina, O.V.; Koshelev, V.B.; Hori, N. \& Carpenter, D.O. (1997). Biochemical and physiological evidence that carnosine is an endogenous neuroprotector against free radicals. Cell Mol Neurobiol, Vol.17,No.2,pp.259-271.

Bunn, H.F. (1991). Pathophysiology of the anemias. Harrison's Principle of Internal Medicine,pp.1514-1518.

Burcham, P.C.; Kaminskas, L.M.; Fontaine, F.R.; Petersen, D.R. \& Pyke, S.M. (2002). Aldehyde-sequestering drugs: tools for studying protein damage by lipid peroxidation products. Toxicology, Vol.181-182,pp.229-236.

Cimen, M.Y. (2008). Free radical metabolism in human erythrocytes. Clin Chim Acta, Vol.390,No.1-2,pp.1-11.

Daugas, E.; Cande, C. \& Kroemer, G. (2001). Erythrocytes: death of a mummy. Cell Death Differ, Vol.8,No.12,pp.1131-1133.

Desouky, O.S. (2009). Rheological and electrical behavior of erythrocytes in patients with diabetes mellitus. Romanian J. Biophys., Vol.19,No.4,pp.239-250.

Eber, S. \& Lux, S.E. (2004). Hereditary spherocytosis--defects in proteins that connect the membrane skeleton to the lipid bilayer. Semin Hematol, Vol.41,No.2,pp.118-141.

Feher, G.; Koltai, K.; Kesmarky, G.; Szapary, L.; Juricskay, I. \& Toth, K. (2006). Hemorheological parameters and aging. Clin Hemorheol Microcirc, Vol.35,No.1-2, pp.89-98.

Gariballa, S.E. \& Sinclair, A.J. (2000). Carnosine: physiological properties and therapeutic potential. Age Ageing, Vol.29,No.3,pp.207-210.

Guyton, A.C. \& Hall, J.E. (2000). Transport of oxygen and carbon dioxide in the blood and body fluids. In: Textbook of medical physiology,pp.463-473, W.B. Saunders. Philadelphia, PA.

Head, D.J.; Lee, Z.E.; Poole, J. \& Avent, N.D. (2005). Expression of phosphatidylserine (PS) on wild-type and Gerbich variant erythrocytes following glycophorin-C (GPC) ligation. Br J Haematol, Vol.129,No.1,pp.130-137.

Hipkiss, A.R. (2005). Glycation, ageing and carnosine: are carnivorous diets beneficial? Mech Ageing Dev, Vol.126,No.10,pp.1034-1039. 
Hipkiss, A.R. \& Brownson, C. (2000). Review: A possible new role for the anti-ageing peptide carnosine. Cell. Mol. Life Sci., Vol.57pp.747-753.

Hipkiss, A.R. \& Chana, H. (1998). Carnosine protects proteins against methylglyoxalmediated modifications. Biochem Biophys Res Commun, Vol.248,No.1,pp.28-32.

Hipkiss, A.R.; Preston, J.E.; Himswoth, D.T.; Worthington, V.C. \& Abbot, N.J. (1997). Protective effects of carnosine against malondialdehyde-induced toxicity towards cultured rat brain endothelial cells. Neurosci Lett, Vol.238,No.3,pp.135-138.

Hipkiss, A.R.; Worthington, V.C.; Himsworth, D.T. \& Herwig, W. (1998). Protective effects of carnosine against protein modification mediated by malondialdehyde and hypochlorite. Biochim Biophys Acta, Vol.1380,No.1,pp.46-54.

Ivanov, I.T. (1999). Low pH-induced hemolysis of erythrocytes is related to the entry of the acid into cytosole and oxidative stress on cellular membranes. Biochim Biophys Acta, Vol.1415,No.2,pp.349-360.

Jacobsen, D.W. (1998). Homocysteine and vitamins in cardiovascular disease. Clin Chem, Vol.44,No.8 Pt 2,pp.1833-1843.

Kohen, R.; Yamamoto, Y.; Cundy, K.C. \& Ames, B.N. (1988). Antioxidant activity of carnosine, homocarnosine, and anserine present in muscle and brain. Proc Natl Acad Sci U S A, Vol.85,No.9,pp.3175-3179.

Lux, S.E. (1979). Dissecting the red cell membrane skeleton. Nature, 281, Vol.5731,No.pp.426429.

Marchesi, V.T. (1985). Stabilizing infrastructure of cell membranes. Annu Rev Cell Biol, Vol.1,No.pp.531-561.

McFarland, G.A. \& Holliday, R. (1994). Retardation of the senescence of cultured human diploid fibroblasts by carnosine. Exp Cell Res, Vol.212,No.2,pp.167-175.

McFarland, G.A. \& Holliday, R. (1999). Further evidence for the rejuvenating effects of the dipeptide L-carnosine on cultured human diploid fibroblasts. Exp Gerontol, Vol.34,No.1,pp.35-45.

Mohandas, N. (1991). The red blood cell membrane.In: Hematology: Basis, Principles and Practice. R. Hoffman, E. J. Benz, S. J. Shattil, B. Furie \& H. J. Cohen,pp.264-269, Churchill-Livingstone.New York.

Mohandas, N. \& Chasis, J.A. (1993). Red blood cell deformability, membrane material properties and shape: regulation by transmembrane, skeletal and cytosolic proteins and lipids. Semin Hematol, Vol.30,No.3,pp.171-192.

Morris, M.B. \& Lux, S.E. (1995). Characterization of the binary interaction between human erythrocyte protein 4.1 and actin. Eur J Biochem, Vol.231,No.3,pp.644-650.

Nam, J.H.; Kim, C.B. \& Shin, S. (2009). The effect of L-carnosine on the rheological characteristics of erythrocytes incubated in glucose media. Korea-Australia Rheology Journal, Vol.21,No.2,pp.103-108.

Nohl, H. \& Stolze, K. (1998). The effects of xenobiotics on erythrocytes. Gen Pharmacol, Vol.31,No.3,pp.343-347.

Pietraforte, D.; Matarrese, P.; Straface, E.; Gambardella, L.; Metere, A.; Scorza, G.; Leto, T.L.; Malorni, W. \& Minetti, M. (2007). Two different pathways are involved in peroxynitrite-induced senescence and apoptosis of human erythrocytes. Free Radic Biol Med, Vol.42,No.2,pp.202-214.

Popel, A.S. \& Johnson, P.C. (2005). Microcirculation and Hemorheology. Annu Rev Fluid Mech, Vol.37,No.,pp.43-69.

Preibisch, G.; Kuffner, C. \& Elstner, E.F. (1993). Biochemical model reactions on the prooxidative activity of homocysteine. Z Naturforsch C, Vol.48,No.1-2,pp.58-62. 
Pribush, A.; Meyerstein, D. \& Meyerstein, N. (2002). Kinetics of erythrocyte swelling and membrane hole formation in hypotonic media. Biochim Biophys Acta, Vol.1558,No.2,pp.119-132.

Prokopiva, V.D.; Bohan, N.A.; Johnson, P.; Abe, H. \& Boldyrev, A.A. (2000). Effect of carnosine and related compounds on the stability and morphology of erythrocytes from alcoholics. Alcohol and Alcoholism, Vol.35,No.1,pp.44-48.

Quinn, P.J.; Boldyrev, A.A. \& Formazuyk, V.E. (1992). Carnosine: its properties, functions and potential therapeutic applications. Mol Aspects Med, Vol.13,No.5,pp.379-444.

Schlussel, E.; Preibisch, G.; Putter, S. \& Elstner, E.F. (1995). Homocysteine-induced oxidative damage: mechanisms and possible roles in neurodegenerative and atherogenic processes. Z Naturforsch C, Vol.50,No.9-10,pp.699-707.

Seidler, N.W.; Yeargans, G.S. \& Morgan, T.G. (2004). Carnosine disaggregates glycated alpha-crystallin: an in vitro study. Arch Biochem Biophys, Vol.427,No.,1,pp.110-115.

Severina, I.S.; Bussygina, O.G. \& Pyatakova, N.V. (2000). Carnosine as a regulator of soluble guanylate cyclase. Biochemistry (Mosc), Vol.65,No.7,pp.783-788.

Smith, C.; Marks, A.D. \& Lieberman, M. (2005). Mark's Basic Medical Biochemistry (edition).Lippincott Williams \& Wilkins, Philadelphia.

Snyder, L.M.; Fortier, N.L.; Trainor, J.; Jacobs, J.; Leb, L.; Lubin, B.; Chiu, D.; Shohet, S. \& Mohandas, N. (1985). Effect of hydrogen peroxide exposure on normal human erythrocyte deformability, morphology, surface characteristics, and spectrinhemoglobin cross-linking. J Clin Invest, Vol.76,No.5,pp.1971-1977.

Tamba, M. \& Torreggiani, A. (1999). Hydroxyl radical scavenging by carnosine and Cu(II)carnosine complexes: a pulse-radiolysis and spectroscopic study. Int J Radiat Biol, Vol.75,No.9,pp.1177-1188.

Telen, M.J. \& Kaufman, R.E. (1999). The mature erythrocyte.In. J. P. Greer \& J. Foerster,pp.217-247, Lippincott Williams \& Wilkins.Philadelphia.

Tse, W.T. \& Lux, S.E. (1999). Red blood cell membrane disorders. Br J Haematol, Vol.104,No.1,pp.2-13.

Vladychenskaya, E.A.; Tyulina, O.V. \& Boldyrev, A.A. (2006). Effect of homocysteine and homocysteic acid on glutamate receptors on rat lymphocytes. Bull Exp Biol Med, Vol.142,No.,1,pp.47-50.

Volpe, E.P. (1993). Blood and circulation.In: Biology and Human Concerns. W. C. Dubuque,pp.253-265, William C Brown Pub

Yapislar, H. \& Aydogan, S. (2011 Vol.85,No.). Effect of carnosine on erythrocyte deformability in streptocotocin induced diabetic rats; relationship between carnosine and nitric oxide. ESCHM Congress. Munich, Germany, June 2011, pp:144.

Yerer, M.B.; Aydogan, B. \& Aydogan, S. (2010). Sodium nitroprusside-induced oxidative damage on erythrocytes: Protective role of carnosine. Series on Biomechanics, Vol.25,No. 1-2,pp.194-198.

Yerer, M.B.; Yapislar, H.; Aydogan, S.; Yalcin, O. \& Baskurt, O. (2004). Lipid peroxidation and deformability of red blood cells in experimental sepsis in rats: The protective effects of melatonin. Clin Hemorheol Microcirc, Vol.30,No.2,pp.77-82.

Yilmaz, F. \& Gundogdu, M. (2008). A critical review on blood flow in large arteries; relevance to blood rheology, viscosity models, and physiologic conditions. KoreaAustralia Rheology J., Vol.20,pp.197-211.

Yuneva, M.O.; Bulygina, E.R.; Galant, S.C.; Kramarenko, G.G.; Stvolinsky, S.L.; Semyonova, M.L. \& Boldyrev, A.A. (1999). Effect of carnosine on age-induced changes in senescence accelerated mice. Journal of Anti-Aging Medicine, Vol.2,No.4,pp.337-342. 


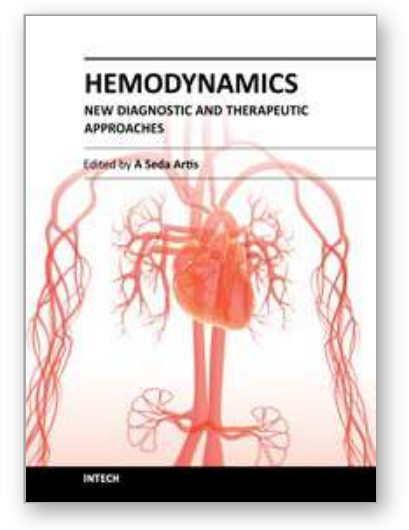

\author{
Hemodynamics - New Diagnostic and Therapeutic Approaches \\ Edited by Dr. A Seda Artis
}

ISBN 978-953-51-0559-6

Hard cover, 156 pages

Publisher InTech

Published online 25, April, 2012

Published in print edition April, 2012

Hemodynamics is study of the mechanical and physiologic properties controlling blood pressure and flow through the body. The factors influencing hemodynamics are complex and extensive. In addition to systemic hemodynamic alterations, microvascular alterations are frequently observed in critically ill patients. The book "Hemodynamics: New Diagnostic and Therapeuric Approaches" is formed to present the up-to-date research under the scope of hemodynamics by scientists from different backgrounds.

\title{
How to reference
}

In order to correctly reference this scholarly work, feel free to copy and paste the following:

A. Seda Artis and Sami Aydogan (2012). Carnosine and Its Role on the Erythrocyte Rheology, Hemodynamics - New Diagnostic and Therapeutic Approaches, Dr. A Seda Artis (Ed.), ISBN: 978-953-51-0559-6, InTech, Available from: http://www.intechopen.com/books/hemodynamics-new-diagnostic-and-therapeuticapproaches/carnosine-and-its-role-on-the-erythrocyte-rheology

\section{INTECH}

open science | open minds

\author{
InTech Europe \\ University Campus STeP Ri \\ Slavka Krautzeka 83/A \\ 51000 Rijeka, Croatia \\ Phone: +385 (51) 770447 \\ Fax: +385 (51) 686166 \\ www.intechopen.com
}

\author{
InTech China \\ Unit 405, Office Block, Hotel Equatorial Shanghai \\ No.65, Yan An Road (West), Shanghai, 200040, China \\ 中国上海市延安西路65号上海国际贵都大饭店办公楼 405 单元 \\ Phone: +86-21-62489820 \\ Fax: +86-21-62489821
}


(C) 2012 The Author(s). Licensee IntechOpen. This is an open access article distributed under the terms of the Creative Commons Attribution 3.0 License, which permits unrestricted use, distribution, and reproduction in any medium, provided the original work is properly cited. 